\title{
APPROXIMATION BY INTEGRAL FUNCTIONS IN THE COMPLEX DOMAIN
}

\author{
BY \\ H. KOBER
}

The approximation by integral functions to functions defined on the real axis $-\infty<x<\infty$ or on its positive part only $(0<x<\infty)$ has been treated by the author in an earlier paper $\left(^{1}\right)$. The present paper deals with the corresponding problem for functions defined on regions $R$ of the extended $z$-plane $(z=x+i y)$. The only case of interest is that in which the point $z=\infty$ lies on the boundary of $R$, as has been shown in the previous paper. Originally the author had intended to treat only the cases where $R$ is a half-plane or a strip. It has turned out, however, that simple results can be obtained for approximation in an angle $\Theta(0<\arg z<\Theta ; \Theta<2 \pi)$. This problem is evidently much more general than that concerning the half-plane, and it is adapted to elucidate results of the above paper. It will be shown that a function $F(z)$, analytic and bounded in the interior of an angle $\Theta$ and satisfying some condition of continuity in the closed domain, can be approximated uniformly by integral functions of finite types of the order $\rho=\pi(2 \pi-\Theta)^{-1}$, and that this is the "best possible" value ( $\$ 2)$. The uniform approximation by integral functions to unbounded functions in an angle $\Theta$, in particular to $z^{r}(0<r<\infty)$, is treated in $\$ 4$; there are, however, rather interesting questions which arise in the general case, but are left open in the present investigation. The approximation by rational functions is known to apply under very restrictive conditions only; it fails, for instance, for the function $z^{r}$.

The approximation to bounded functions in the half-plane is treated in $\S 3$, that in the strip in $\$ 5$. For the half-plane, extensive use is made of the fundamental theory of the class $\mathfrak{S}_{p}\left({ }^{2}\right)$, developed by E. Hille and J. D. Tamarkin; some of the results deduced for the strip can be regarded as an extension of this theory. The investigation of the strip starts from Theorem 13. Its proof and that of some other results is given as an appendix. Incidentally results

Presented to the Society, April 29, 1944; received by the editors October 28, 1943.

(1) Trans. Amer. Math. Soc. vol. 54 (1943). References to this paper are indicated by F. There is an error in equation (2.1)F, which should read

$$
W(r)=\int_{0}^{2 \pi n} \prod_{j=1}^{n} f_{i}\left(r e^{i \phi}\right)^{p j} d \phi .
$$

Unfortunately, in my manuscript I have written $\sum$ instead of $\prod$.

(2) Fund. Math. vol. 25 (1935) pp. 329-352, $1 \leqq p<\infty$. For $0<p<1$ see T. Kawata, Jap. J. Math. vol. 13 (1936) pp. 421-430. $\mathfrak{S}_{p}(0<p \leqq \infty)$ is the set of functions $F(z)$ which, for $0<y<\infty$, are analytic and such that $|F(x+i y)|_{p} \leqq M$, where $M$ is independent of $y$. For the notation $|F(x+i y)|_{p}$ see the end of this section. 
due to G. H. Hardy, A. E. Ingham, and G. Pólya $\left({ }^{3}\right)$ are generalized.

Applications of results of this paper and the one mentioned above will be made in $\S 6$, in particular to deduce a new theorem on approximation by rational functions in a strip. Some auxiliary results are given in $\$ 1$, among them a theorem which is a supplement to Theorem $3(\mathrm{~b}) \mathrm{F}$.

The symbol $|f(x+i y)|_{p}$ will be used to denote

$$
\left(\int_{-\infty}^{\infty}|f(x+i y)|^{p} d x\right)^{1 / p} \text { or } \underset{-\infty<x<\infty}{\text { ess.u.b. }}|f(x+i y)|
$$

for $0<p<\infty$ or $p=\infty$, respectively; in this notation the first variable which occurs is that of integration. Finite positive constants are denoted by $A, A^{\prime}, A^{\prime \prime}, \cdots, B, B^{\prime}, B^{\prime \prime}, \cdots$. Throughout this paper the function $F(z)$ which is to be approximated is required not to reduce to a constant.

\section{§1. Some AUXILIARY RESUlts}

\subsection{Functions of order one and finite type.}

Lemma 1. Let $\kappa(z) \in G_{\alpha}(0<\alpha<\infty)\left({ }^{4}\right)$ and $\kappa(x) \in L_{p}(-\infty, \infty)(0<p<\infty)$. Then $\kappa(x+i y) e^{-\alpha|y|} \rightarrow 0$ as $|x| \rightarrow \infty$, uniformly for $-\infty<y<\infty$, and thus $\kappa(x+i y) \rightarrow 0$ as $|x| \rightarrow \infty$, uniformly in any finite interval of $y$.

It will suffice to take $y>0$. By Lemma $2^{\prime}(\mathrm{b}) \mathrm{F}, \quad|\kappa(x+i \tilde{y})|_{p}$ $\leqq|\kappa(x)|_{p} \exp (\alpha|\tilde{y}|)$. Taking $\tilde{y}=y^{\prime}-1$, we have $\left|\exp \left(i \alpha x-\alpha y^{\prime}\right) \kappa\left(x+i y^{\prime}-i\right)\right|_{p}$ $\leqq e^{\alpha}|\kappa(x)|_{p}$ for $y^{\prime} \geqq 0$. Hence the function $\exp (i \alpha z) \kappa(z-i)\left(z=x+i y^{\prime}\right)$ belongs to $\mathfrak{W}_{p}$ and tends, therefore $\left.{ }^{5}\right)$, to zero as $|x| \rightarrow \infty$, uniformly for $y^{\prime} \geqq \delta$ whenever $\delta>0$. Taking $y^{\prime}=y+1$ and $\delta=1$, we have proved the lemma.

\subsection{Functions of finite order and type.}

LEMMA 2. Let (i) $F(z)$ be an integral function of the type $\alpha(0 \leqq \alpha<\infty)$ of the order $\rho(1 / 2<\rho<\infty)$, let (ii) the contour $C$ consist of two straight lines starting at the origin and forming the angles $\Theta=2 \pi-\pi \rho^{-1}$ and $\Gamma=\pi \rho^{-1}$ between them, let $F(z)$ be bounded on $C$ with upper bound $A$, and let (iii) $F(z)$ be bounded in the angle $\Theta$. Then, for any $z,|F(z)| \leqq A \exp \left(\alpha|z|^{\rho}\right)$. When $1 / 2<\rho \leqq 1$ the condition (iii) is to be omitted.

Proof. For $\Theta=\pi(\rho=1)$ the result is well known $\left({ }^{6}\right)$. By a PhragménLindelöf $\left({ }^{7}\right)$ theorem, $|F(z)| \leqq A$ in the angle $\Theta$ for $1 / 2<\rho<1$ and, by (iii), for $\rho>1$. We may take $\arg z=(\Theta-\pi) / 2$ and $\arg z=(3 \pi-\Theta) / 2$ as the two

(3) Proc. Royal Soc. London, Ser. A vol. 113 (1927) pp. 542-569.

$\left.{ }^{4}\right) G_{\alpha}$ is the set of integral functions $F(z)$ which, for any $\epsilon>0$, satisfy an inequality $|F(z)|<A_{\epsilon} \exp \{(\alpha+\epsilon)|z|\}$. Obviously $G_{\beta} \subset G_{\alpha}$ whenever $0 \leqq \beta<\alpha$; trivially any polynomial belongs to $G_{0}$.

(5) Hille-Tamarkin, loc. cit., Lemma 2.4.

(6) G. Pólya and G. Szegö, Aufgaben und Lehrsätze aus der Analysis, vol. 2, Berlin, 1925, p. 36, no. 202. A shorter proof is given in $\$ 2 \mathrm{~F}$.

(7) See, for instance, E. C. Titchmarsh, The theory of functions, Oxford, 1932, 5.61. 
lines forming the angle $\Gamma$; it is bisected by the positive part of the imaginary axis which we denote by $L$. Given $\epsilon>0$, the function

$$
G(z)=F(z) \exp \left\{(\alpha+2 \epsilon) z^{\rho} e^{i \pi-i \pi \rho / 2}\right\}
$$

is analytic in $\Gamma$, its modulus is less than $A^{\prime} \exp \left\{(2 \alpha+3 \epsilon)|z|^{\rho}\right\}$ in $\Gamma$ and is not greater than $A$ on $C$. On $L$, we have

$$
|G(z)| \leqq|F(z)| \exp \{-(\alpha+2 \epsilon)|z| \rho\} \leqq A^{\prime \prime} e^{-\epsilon|z| \rho},
$$

which tends to zero as $|z| \rightarrow \infty$. Applying the above mentioned theorem to $G(z)$ in both angles formed by $L$ and $C$, we deduce that $G(z)$ is bounded in both angles and, therefore, in $\Gamma$. But $|G(z)| \leqq A$ on $C$; therefore, by the same Phragmén-Lindelöf theorem, $|G(z)| \leqq A$ in $\Gamma$. Hence $|F(z)| \leqq A$ exp $\{(\alpha+2 \epsilon)|z| \rho\}$ in $\Gamma$ and, therefore, in the whole plane. Taking $\epsilon \rightarrow 0$ we arrive at the required result.

Remark. If $z$ lies in $\Gamma$, and if $\psi$ is the angle between the line joining the origin to $z$ and one of the two lines bounding $\Gamma(0<\psi<\Gamma)$, then

$$
|F(z)| \leqq A \exp \left\{\alpha|z|^{\rho} \sin \rho \psi\right\}\left(^{8}\right) \text {. }
$$

This follows from (1.21), taking $z=r \exp \{i(\Theta-\pi+2 \psi) / 2\}$.

By the Liouville theorem, $F(z)$ reduces to a constant when $\alpha=0$.

1.3. Induced convergence.

LEMma $3(\mathrm{a})$. Let the $F_{n}(z)(n=1,2, \cdots)$ be integral functions of a fixed type $\alpha$ of the order $\rho(0<\alpha<\infty, 1 / 2<\rho \leqq 1)$, let $\Theta$ and $C$ be defined as in Lemma 2 and let, uniformly on $C, F_{m}(z)-F_{n}(z) \rightarrow 0$ as $m, n \rightarrow \infty$. Then there is an integral function $F(z)$ such that, for any $\epsilon>0,|F(z)|<A_{\epsilon} \exp \{(\alpha+\epsilon)|z| \rho\}$ and that, uniformly in the angle $\Theta, F_{n}(z) \rightarrow F(z)$ as $n \rightarrow \infty$.

(b) For $1<\rho<\infty$ the result holds under the additional condition that the functions $F_{1}(z)-F_{n}(z)(n=2,3, \cdots)$ should be bounded in $\Theta$.

Proof. The functions $F_{1, n}(z)=F_{1}(z)-F_{n}(z)(n=2,3, \cdots)$ are bounded on $C$, uniformly with respect to $z$ and $n$. By Lemma 2 , there is a constant $A$ independent of $n$ such that

$$
\left|F_{1, n}(z)\right| \leqq A \exp \left\{\alpha|z|^{\rho}\right\} \quad(1 / 2<\rho<\infty) .
$$

By the argument used in the proof of Lemma $3 \mathrm{~F}$, we deduce that the $F_{1, n}(z)$ converge to an integral function $\Phi(z)$ satisfying (1.31), uniformly in any finite domain and uniformly on $C$. Hence $F(z)=F_{1}(z)-\Phi(z)$ is the required function.

1.4. Functions bounded in sectors.

LeMma 4. Let $\kappa(z)$ be an integral function of the type $b(0<b<\infty)$ of the order $\rho(1 \leqq \rho<\infty)$, bounded in the domain $D$ defined by $D=D_{1}+D_{2}$,

(8) It can be shown that the result holds for the limiting case $\rho=1 / 2$. If, therefore, $F(z)$ is an integral function of the type $\alpha$ of the order $1 / 2$ and $|F(x)| \leqq A$ for $0<x<\infty$, then $|F(z)|$ $\leqq A \exp \left\{\alpha(|z| / 2-x / 2)^{1 / 2}\right\}$. 


$$
D_{1}: \quad|\arg z| \leqq \pi / 2-\pi / 2 \rho, \quad D_{2}: \quad|\arg z-\pi| \leqq \pi / 2-\pi / 2 \rho,
$$

with upper bound $A$. Then $|\kappa(z)| \leqq A \exp \left(b|y|^{\rho}\right)$.

As in the proof of Lemma 2, we deduce that, for $|\arg z-\pi / 2| \leqq \pi(2 \rho)^{-1}$,

$$
\begin{aligned}
|\kappa(z)| \leqq A \exp \{b|z| \rho \cos \rho(\pi / 2-\phi)\} \\
\quad(\phi=\arg z ;|\phi-\pi / 2| \leqq \pi / 2 \rho) .
\end{aligned}
$$

We have $y=|z| \sin \phi>0$, and $\sin ^{\rho} \phi \geqq \cos \rho(\pi / 2-\phi)$, by the inequality $\cos \rho \lambda \leqq \cos ^{\rho} \lambda\left(\rho \geqq 1,0 \leqq \lambda \leqq \pi(2 \rho)^{-1}\right)$. Hence from (1.41) and from the hypotheses we deduce that $|\kappa(z)| \leqq A$ exp $(b|y| \rho)$ for $y \geqq 0$. In a similar way the result is shown for $y<0$, which completes the proof.

LEMMA 5. If $l$ is any positive integer, then there are even functions $h(z)$ such that $z^{i} h(z)(j=0,1, \cdots, 2 l)$ satisfies the hypotheses of Lemma 4.

Without loss of generality we may take $b=1$. The Mittag-Leffler function

$$
E_{1 / \rho}(z)=\sum_{n=0}^{\infty} \frac{z^{n}}{\Gamma\left(1+\rho^{-1} n\right)}
$$

is of the type unity of the order $\rho$, and is bounded for $|\arg z-\pi| \leqq \pi-\pi(2 \rho)^{-1}$ when $1 / 2 \leqq \rho<\infty$. Now

$$
h(z)=z^{-2 l}\left\{E_{1 / \rho}\left(i l^{-1 / \rho} z\right)+E_{1 / \rho}\left(-i l^{-1 / \rho} z\right)-2\right\}^{l} \quad(\rho \geqq 1)
$$

is the required function. For $E_{1 / \rho}(i z)$ is bounded for $\pi(2 \rho)^{-1}-\pi / 2 \leqq \arg z$ $\leqq 3 \pi / 2-\pi(2 \rho)^{-1}$, while $E_{1 / \rho}(-i z)$ is bounded for $\pi(2 \rho)^{-1}-3 \pi / 2 \leqq \arg z \leqq \pi / 2$ $-\pi(2 \rho)^{-1}$ and, therefore, for $\pi / 2+\pi(2 \rho)^{-1} \leqq$ arg $z \leqq 5 \pi / 2-\pi(2 \rho)^{-1}$. then

LEMma 6. If $\rho \geqq 1$ and $H(z)=z^{i} h(z)(0 \leqq j \leqq 2 l-2)$, defining $h(z)$ by (1.43),

$$
\int_{-\infty}^{\infty}|H(t-z)| d t \leqq A \exp (|y| \rho) \quad(A=A(l, \rho)) .
$$

Putting $t=u+x$, the integral on the left takes the form $\int_{-\infty}^{\infty}|H(u-i y)| d u$. When $\rho=1$, then, by Lemma $2^{\prime}(\mathrm{b}) \mathrm{F},|H(u-i y)|_{1} \geqq \exp (|y|)|H(u)|_{1}$. Let now $\rho>1$. For $|y|<1$, the integral is certainly majorized by a constant $A^{\prime}$, since $H(x+i y)$ and $x^{2} H(x+i y)$ are bounded in the strip $|y| \leqq 1,|x|<\infty$. For $|y| \geqq 1$, we have

$$
\begin{aligned}
&\left(t^{2}+1\right)^{l-j / 2}|H(t-i y)| \leqq|t-i y|^{2 l}|h(t-i y)| \leqq A^{\prime \prime} e^{|y|^{\rho}}, \\
& \int_{-\infty}^{\infty}|H(t-i y)| d t \leqq A^{\prime \prime} e^{|y|^{\rho}} \int_{-\infty}^{\infty}\left(t^{2}+1\right)^{j / 2-l} d t \leqq A^{\prime \prime \prime} e^{|y|^{\rho}},
\end{aligned}
$$

which proves the lemma.

1.5. An approximation theorem concerning functions of $L_{\infty}(-\infty, \infty)$. 
Theorem 1. Let $F(t) \in L_{\infty}(-\infty, \infty)$, that is to say, let $F(t)$ be measurable and essentially bounded in $(-\infty, \infty)$. Then there are functions $g_{\alpha}(z) \in G_{\alpha}$ $(0<\alpha<\infty)$ such that, for almost all $x$ in $(-\infty, \infty), g_{\alpha}(x) \rightarrow F(x)$ as $\alpha \rightarrow \infty$.

Let $\kappa(z) \in G_{1}$ and such that both $\kappa(t)$ and $t \kappa^{\prime}(t)$ belong to $L_{1}(-\infty, \infty)$, and that $\int_{-\infty}^{\infty} \kappa(t) d t=1$ (see (6.3)F), and take

$$
g_{\alpha}(x)=\alpha \int_{-\infty}^{\infty} \kappa(\alpha t) F(t+x) d t
$$

Then $\left|g_{\alpha}(x)\right| \leqq|\kappa(t)|_{1}|F(t)|_{\infty}$, and

$$
g_{\alpha}(x)-F(x)=\int_{-\infty}^{\infty} \kappa(t)\{F(x+t / \alpha)-F(x)\} d t .
$$

Let $x$ be any point of the "Lebesgue set" of $F(x)$, and

$$
H_{\alpha}(t)=\int_{0}^{t}\{F(x+u / \alpha)-F(x)\} d u=\alpha \int_{0}^{t / \alpha}\{F(x+u)-F(x)\} d u .
$$

Then $H_{\alpha}(t)=o(t)$ as $t / \alpha \rightarrow 0$. Integrating by parts, from (1.52) we deduce that

$$
F(x)-g_{\alpha}(x)=\int_{-\infty}^{\infty} \kappa^{\prime}(t) H_{\alpha}(t) d t
$$

taking into consideration that $t \kappa^{\prime}(t) \rightarrow 0$ as $t \rightarrow \pm \infty$ and that $\left|H_{\alpha}(t)\right|$ $\leqq 2|t||F(t)|_{\infty}$. Given $\epsilon>0$, we put

$$
F(x)-g_{\alpha}(x)=\left(\int_{-\infty}^{-M}+\int_{-M}^{M}+\int_{M}^{\infty}\right) \kappa^{\prime}(t) H_{\alpha}(t) d t=I_{1}+I_{2}+I_{3}
$$

and fix $M$ so that, for any $\alpha>0,\left|I_{1}+I_{3}\right| \leqq \epsilon / 2$. Taking $B=\int_{-M}^{M}\left|t \kappa^{\prime}(t)\right| d t$, we can find a $\delta$ such that

$$
\left|I_{3}\right| \leqq B \max _{-M \leqq t \leqq M}\left|t^{-1} H_{\alpha}(t)\right|<\epsilon / 2 \quad \text { for } \quad|t / \alpha| \leqq \delta \quad(|t| \leqq M) .
$$

Hence $\left|F(x)-g_{\alpha}(x)\right| \leqq \epsilon$ for $\alpha \geqq M \delta^{-1}$, therefore $g_{\alpha}(x) \rightarrow F(x)$, as $\alpha \rightarrow \infty$, at any point $x$ of the Lebesgue set. This proves the theorem.

\section{§2. ON FUNCTIONS ANALYTIC AND BOUNDED IN AN ANGLE}

\subsection{Order of the approximating function.}

Theorem 2. Let $0<\Theta<2 \pi$ and $F(z)\left(z=x+i y=|z| e^{i \phi}\right)$ be analytic in the interior of the angle $\Theta(0<\phi<\Theta)$, and let, for any $\epsilon>0, F(z)$ be bounded and uniformly approximated by integral functions $g_{n}(z)\left(n=1,2, \cdots ; g_{n}(z)\right.$ independent of $\epsilon)$ of order $\rho$ in the domain $\epsilon \leqq \phi \leqq \Theta-\epsilon, 0 \leqq|z|<\infty$. Then $\rho \geqq \pi(2 \pi-\Theta)^{-1}$. 
Proof. Suppose $\rho$ to be smaller than $\pi(2 \pi-\Theta)^{-1}$; then there is an $\epsilon>0$ such that $\rho+\epsilon<\pi(2 \pi+2 \epsilon-\Theta)^{-1}$. Each of the functions $g_{n}(z)$ is bounded for $\epsilon \leqq \phi \leqq \Theta-\epsilon$. In the remaining angle $\Theta-\epsilon<\phi<2 \pi+\epsilon$ we have $g_{n}(z)$ $=O\left\{\exp \left(|z|^{\rho+\varepsilon}\right)\right\}$ as $|z| \rightarrow \infty$, while $g_{n}(z)$ is bounded on the two straight lines forming this angle. It is less than $\pi(\rho+\epsilon)^{-1}$, therefore $g_{n}(z)$ is bounded in this angle, again by a Phragmen-Lindelöf theorem. Hence, by the Liouville theorem, $g_{n}(z)$ reduces to a constant. So does $F(z)$, which is a contradiction.

Remark. If $F(z)$ is not bounded and $\rho<\pi(2 \pi-\theta)^{-1}$, then $F(z)$ is an integral function of order $\rho$.

THEOREM 3. Let $F(z)$ and the $g_{n}(z)$ satisfy the conditions of Theorem 2. Given any $\rho^{\prime}>\rho$, there is a set $f_{n}(z)$ of integral functions of order $\rho^{\prime}$ which approximate to $F(z)$, as $n \rightarrow \infty$. uniformly in the domain $\epsilon \leqq \phi \leqq \Theta-\epsilon$ for any $\epsilon>0$.

We have to find a single integral function $H(z)$ of order $\rho^{\prime}$ which is bounded for $0 \leqq \phi \leqq \Theta$. We can take, for instance, $H(z)=E_{1 / \rho^{\prime}}\left\{z \exp \left(i \pi\left(2 \rho^{\prime}\right)^{-1}\right)\right\}$. Then $\left\{f_{n}(z)\right\}=\left\{g_{n}(z)+n^{-1} H(z)\right\}(n=1,2, \cdots)$ is the required set. Evidently the result holds dropping the condition that $F(z)$ should be bounded.

Remark. The theorems remain true if $\epsilon$ is replaced by zero.

2.2. Best order. By Theorem 3 there exists a lower bound of the orders of the integral functions which approximate to an analytic bounded function in the angle $\Theta$. By Theorem 2, this lower bound is not smaller than $\pi(2 \pi-\Theta)^{-1}$. This minimum value is in fact attained.

THEOREM 4. Let $0<\Theta<2 \pi$ and (i) $F(z)(z=x+i y)$ be analytic and bounded in the angle $|\arg z-\pi / 2|<\Theta / 2$, with limit-function $F(\zeta)(z \rightarrow \zeta$, arg $\zeta=\pi / 2 \pm \Theta / 2)$, (ii) $F(z)$ be uniformly continuous on the lines bounding this angle, or (ii)' $F\left(z^{2}\right)$ be uniformly continuous on the two lines $\arg z=(\pi-\Theta) / 4$ and $\arg z=(\pi+\Theta) / 4$ $(0 \leqq|z|<\infty)$, for $\pi \leqq \Theta<2 \pi$ or $0<\Theta<\pi$, respectively. Then there is a set of integral functions $g_{\alpha}(z)(0<\alpha<\infty)$ and a constant $A$, independent of $\alpha$, such that

$$
\left|g_{\alpha}(z)\right| \leqq A \exp \left(|\alpha z|^{\rho}\right), \quad \rho=\pi(2 \pi-\Theta)^{-1},
$$

and that $g_{\alpha}(z) \rightarrow F(z)$, as $\alpha \rightarrow \infty$, uniformly in the angle $|\arg z-\pi / 2|<\Theta / 2$.

For the border line case $\Theta=0(\rho=1 / 2)$ see Theorem $3(a, b) F$.

We note that the existence of the limit function $F(\zeta)$ at almost all points $\zeta$ is a consequence of the regularity and boundedness of $F(z)$ in the interior of the angle $\Theta$ and that, therefore, $F(z)$ is defined for $|\arg z-\pi / 2| \leqq \Theta$, $0 \leqq|z|<\infty$ (compare $\$ 2.4$ ). The conditions (ii) and (ii)' are not necessary, except for $\Theta=\pi$ and for the limiting case $\Theta=0$ (Theorem 3(b)F). Take, for instance, $F(z)=E_{1 / p}(i z)$. Possibly both (ii) and (ii)' can be replaced by the weaker condition (ii) $)^{\prime \prime}: F\left(z^{1 / p}\right)$ is uniformly continuous on the two lines $\arg z=(2 \rho)^{-1}(\pi \mp \Theta)$.

We need some lemmas. 
LeMma 7. Let $k$ be a non-negative integer, let $F(t)$ be defined for almost all $t$ in $(-\infty, \infty)$, measurable and bounded on any finite interval and $O\left(|t|^{k}\right)$ as $|t| \rightarrow \infty$; let $\rho \geqq 1, l \geqq k / 2+1$ and $h(z)$ be defined by (1.43). Then

$$
g_{\alpha}(z)=\alpha \int_{-\infty}^{\infty} h(\alpha t-\alpha z) F(t) d t
$$

is an integral function and satisfies the inequality

$$
\left|g_{\alpha}(z)\right| \leqq A\left(1+|z|^{k}+\alpha^{-k}\right) \exp \left(|\alpha y|^{\rho}\right) \quad(A=A(k, \rho)) .
$$

First we take $k=0$, that is $F(t) \in L_{\infty}(-\infty, \infty)$. Then

$$
\left|g_{\alpha}(z / \alpha)\right| \leqq \alpha|F(t)|_{\infty} \int_{-\infty}^{\infty}|h(\alpha t-z)| d t=|F(t)|_{\infty} \int_{-\infty}^{\infty}|h(t-z)| d t .
$$

Using Lemma 6 we arrive at (2.21). Now we take $k>0$. Using Lemma 6 again the proof is completed in a way similar to that of Lemma $5 F$.

\subsection{Properties of boundedness.}

LEMMA 8. If $F(z)$ satisfies the condition (1) of Theorem 4 , for $\Theta \geqq \pi$, then the functions $g_{\alpha}(z)(0<\alpha<\infty)$, defined by (2.22), are bounded for $|\arg z-\pi / 2|$ $\leqq \Theta / 2$, uniformly with respect to $\alpha$.

First we take $0<\arg z<\pi$. Let $z$ be any fixed point in the upper halfplane and $B$ the upper bound of $F(z)$ in the angle. We draw a small semicircle with centre at the origin, apply the Cauchy theorem and observe that $h(\tau-z)(\tau-z)^{2}$ is bounded as $|\tau| \rightarrow \infty$, uniformly for $0 \leqq \Im(\tau) \leqq y$. Thus we can move the path of integration upwards, and so

$$
\left|g_{\alpha}(z)\right|=\alpha\left|\int_{i y-\infty}^{i \nu+\infty} h(\alpha t-\alpha z) F(\tau) d \tau\right| \leqq B \int_{-\infty}^{\infty}|h(t)| d t .
$$

Hence the $g_{\alpha}(z)$ are bounded for $0<y<\infty$, uniformly with respect to $\alpha$.

Now we take $z=u e^{i \phi}$ where $-\infty<u<\infty$ and $|\phi|<(\Theta-\pi) / 2$. Using the Cauchy theorem again, we turn the line of integration by the angle $\phi$, and so

$$
\begin{aligned}
&\left|g_{\alpha}(z)\right|=\alpha\left|\lim _{T \rightarrow \infty} \int_{-T e^{i \phi}}^{T e^{i \phi}} h(\alpha \tau-\alpha z) F(\tau) d \tau\right| \leqq B \alpha \int_{-\infty}^{\infty}\left|h\left\{\alpha e^{i \phi}(t-u)\right\}\right| d t, \\
&\left|g_{\alpha}(z)\right| \leqq B \int_{-\infty}^{\infty}\left|h\left(t e^{i \phi}\right)\right| d t .
\end{aligned}
$$

The function $h(\tau)$ is certainly bounded for $|\tau| \leqq 1$, while $h(\tau)=O\left(|\tau|^{-2}\right)$, as $|\tau| \rightarrow \infty$, uniformly for $|\arg \tau|<(\Theta-\pi) / 2$. This completes the proof for the case $\pi<\Theta<2 \pi$. The proof for $\Theta=\pi$ is left to the reader.

2.4. Proof of the theorem for $\pi<\Theta<2 \pi$. For $\Theta=\pi$, see $\$$. 
Let $\kappa(z)=a h(z)$ where $h(z)$ is defined by (1.43) and $a$ is fixed so that $\int_{-\infty}^{\infty} \kappa(t) d t=1$. Then

$$
\lim _{T \rightarrow \infty} \int_{-T e^{i \psi}}^{T e^{i \psi}} k(\tau) d \tau=1 \quad\left(\tau=t e^{i \psi},-\infty<t<\infty\right),
$$

whenever $|\psi| \leqq(\Theta-\pi) / 2$. The function $g_{\alpha}(z)$ is defined by (2.22), replacing $h(z)$ by $\kappa(z)$, and $z$ is supposed to lie on the straight line arg $z=(\pi-\Theta) / 2=\phi$ $(0 \leqq|z|<\infty)$. First we show that $g_{\alpha}(z)$ can be represented in the form

$$
g_{\alpha}(z)=\alpha e^{i \phi} \int_{-\infty}^{\infty} \kappa\left\{\alpha e^{i \phi}(t-|z|)\right\} F\left(t e^{i \phi}\right) d t .
$$

For fixing $z=|z| e^{i \phi}$ and $\alpha$, and turning the path of integration in (2.22), we have $g_{\alpha}(z)=\alpha e^{i \psi} \int_{-\infty}^{\infty} k\left(\alpha t e^{i \psi}-\alpha z\right) F\left(t e^{i \psi}\right) d t(\phi<\psi \leqq 0)$. Given $\epsilon>0$ we can find a number $M$ such that

$$
\alpha\left(\int_{-\infty}^{-M}+\int_{M}^{\infty}\right)\left|\kappa\left(\alpha t e^{i \psi}-\alpha z\right)\right| d t<\epsilon B^{-1},
$$

uniformly with respect to $\psi$ for $\phi \leqq \psi \leqq 0$ as we can easily see from a figure. using Lemma 5. By the Lebesgue convergence theorem, we have

$$
\int_{-M}^{M} \kappa\left(\alpha t e^{i \psi}-\alpha z\right) F\left(t e^{i \psi}\right) d t \rightarrow \int_{-M}^{M} \kappa\left(\alpha t e^{i \phi}-z\right) F\left(t e^{i \phi}\right) d t
$$

as $\psi \rightarrow \phi$, which, together with (2.43), finally gives (2.42).

Using (2.41), we deduce from (2.42) that

$$
\begin{array}{r}
g_{\alpha}(z)-F(z)=e^{i \phi} \int_{-\infty}^{\infty} \kappa\left(t e^{i \phi}\right)\left(F\left\{e^{i \phi}(t / \alpha+|z|)\right\}-F\left(e^{i \phi}|z|\right)\right) d t \\
\quad(0 \leqq|z|<\infty) .
\end{array}
$$

By the conformal transformation $z=w^{\Theta / \pi} \exp (i \pi / 2-i \Theta / 2)$, we deduce from (i) and (ii) that $F(z)$ is continuous for $|\arg z-\pi / 2| \leqq \Theta / 2,0 \leqq|z|<\infty$. Hence. $F(\tau)\left(\tau=v e^{i \phi}\right)$ is uniformly continuous for $\delta \leqq v<\infty(\delta>0)$. Using a familiar argument, we deduce that, uniformly on the line $\arg z=(\pi-\Theta) / 2=\phi$, $g_{\alpha}(z) \rightarrow F(z)$ as $\alpha \rightarrow \infty$. The same result applies to the line arg $z=(\pi+\Theta) / 2$ $(0 \leqq|z|<\infty)$.

In consequence of the hypothesis and of Lemma 8, the function $g_{\alpha}(z)-F(z)$ is bounded for $|\arg z-\pi / 2|<\Theta / 2$, We can now employ a Phragmén-Lindelöf theorem. Thus we have, uniformly for $|\arg z-\pi / 2|<\Theta / 2$,

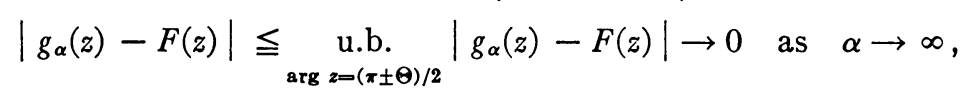

which completes the proof.

2.5. The case $0<\Theta<\pi$. The function $F\left(z^{2}\right)$ is analytic for $\mid \arg z-\pi / 4$ $<\Theta / 4$ and, defining $F(z)=F\left(z e^{-2 i \pi}\right)$ for $|\arg z+3 \pi / 2| \leqq \Theta / 2$, for $|\arg z+3 \pi / 4|$ 
$<\Theta / 4, F\left(z^{2}\right)$ is uniformly continuous on the two lines $z=u$ exp $(i \pi / 4 \pm i \Theta / 4)$ $(-\infty<u<\infty)$ which form both the above angles between them. By the substitution $\Theta^{\prime}=\pi+\Theta / 2, \tilde{z}=z e^{-i \pi / 4}$ the regions concerned become $|\arg \tilde{z}|$ $<\left(\Theta^{\prime}-\pi\right) / 2$ and $|\arg \tilde{z}+\pi|<\left(\Theta^{\prime}-\pi\right) / 2$. The function

$$
Q_{\alpha}(\tilde{z})=\alpha^{1 / 2} \int_{-\infty}^{\infty} \kappa\left\{\alpha^{1 / 2}(t-\tilde{z})\right\} F\left\{\left(t e^{i \pi / 4}\right)^{2}\right\} d t
$$

has the following properties:

(I) It is an integral function, and $\left|Q_{\alpha}(z)\right|<A \exp \left(\left|z \alpha^{1 / 2}\right| \rho^{\prime}\right)$ $\left(\rho^{\prime}=\pi /\left(2 \pi-\Theta^{\prime}\right)\right)$. This follows from Lemma 7 .

(II) $Q_{\alpha}(\tilde{z}) \rightarrow F\left(\tilde{z}^{2} e^{i \pi / 2}\right)$ as $\alpha \rightarrow \infty$, uniformly for $|\arg \tilde{z}|<\left(\Theta^{\prime}-\pi\right) / 2$ and $|\arg \tilde{z}+\pi|<\left(\Theta^{\prime}-\pi\right) / 2$. This can be seen by arguments used in $\S \S 2.3$ and 2.4 .

(III) $Q_{\alpha}(z)$ is an even function; for $\kappa(\tilde{z})$ is even.

Therefore $P_{\alpha}(w)=Q_{\alpha}\left(w^{1 / 2}\right)$ is an integral function, and $\left|P_{\alpha}(w)\right|<A \exp (|\alpha w| \rho)$, where $\rho=\rho^{\prime} / 2=\pi\left(2 \pi-\Theta^{\prime}\right)^{-1} / 2=\pi(2 \pi-\Theta)^{-1}$. Taking $w=\widetilde{z}^{2}$, from (II) we have $P_{\alpha}(w) \rightarrow F\left(w e^{i \pi / 2}\right)$ as $\alpha \rightarrow \infty$, uniformly for $\mid$ arg $w \mid<\Theta^{\prime}-\pi=\Theta / 2$. Finally, putting $g_{\alpha}(z)=P_{\alpha}\{z \exp (-i \pi / 2)\}$, we have $g_{\alpha}(z) \rightarrow F(z)$ uniformly for $|\arg z-\pi / 2|<\Theta / 2$, and $\left|g_{\alpha}(z)\right|<A \exp \left(|\alpha z|^{\rho}\right)$. This completes the proof.

2.6. The problem of best approximation. By means of Lemma 3, we can prove

TheOREM 5. Let $F(z)$ be defined for $|\arg z-\pi / 2|<\Theta / 2$, whether it be analytic or not, and bounded in this angle, and let $b>0$ be fixed. Then in the set $G$, consisting of the integral functions of the type $b$ of the order $\rho=\pi(2 \pi-\Theta)^{-1}$ or of smaller type or order, there is at least one function $g(z)$ such that, for any $h(z) \in G$,

$$
\underset{|\arg z-\pi / 2|<\theta / 2}{u . b}|F(z)-g(z)| \leqq \underset{|\arg z-\pi / 2|<-b_{i / 2}}{u}|F(z)-h(z)| .
$$

\subsection{Approximation in regions different from the interior of an angle.}

THEOREM 6. Let (i) $R$ be a region extending to infinity, bounded by a Jordan curve $C\left(^{9}\right)$, and let all the points of $R$ lie in the interior of an angle $\Theta(0<\Theta<2 \pi)$. Let (ii) $F(z)$ be analytic and bounded in $R$ and (iii) its limit function be continuous everywhere on $C$, including infinity. Then $F(z)$ can be approximated by integral functions of order $\rho=\pi(2 \pi-\Theta)^{-1}\left({ }^{10}\right)$ uniformly in $R($ or $R+C)$.

${ }^{(9)}$ Here $C$ is a Jordan curve in the general sense, that is to say, it is mapped on an ordinary finite closed Jordan curve $\Gamma$ by any substitution $w=\left(z-z_{0}\right)^{-1}$ where $z_{0}$ does not lie on $C$, exterior to $R+C$, say. The function $F(z)$ is said to be continuous everywhere on $C$, including infinity, when it tends to a finite limit $L$, as $z$ moves towards the point at infinity along $C$. Then $A(w)=F\left(z_{0}+w^{-1}\right)$ is continuous on the curve $\Gamma$ and, by (ii), analytic and bounded in its interior $R^{\prime}$, therefore continuous in the closed domain $R^{\prime}+\Gamma$. Hence, uniformly in $R+C, F(z) \rightarrow L$ as $|z| \rightarrow \infty$.

$\left.{ }^{(10}\right)$ Among the functions approximating to $F(z)$ there may occur integral functions of order smaller than $\rho$. By the argument of Theorem 3, they can be replaced by functions of order $\rho$. This applies to Theorems $7,7^{\prime}, 10$. 
Let the angle $\Theta$ be formed by the lines $\arg z=\pi / 2-\Theta / 2$ and $\arg z=\pi / 2$ $+\Theta / 2$. It is known that, uniformly on $C+R, F(z)$ can be approximated by rational functions whose only singularity lies outside $C+R$, at the point $z=-i$, say. Hence $\left({ }^{11}\right)$, given $\epsilon>0$, there are numbers $a_{k}(k=0,1, \cdots, n)$ such that

$$
\left|F(z)-\sum_{k=0}^{n} a_{k}(z+i)^{-k}\right| \leqq \epsilon,
$$

uniformly in $C+R$. By Theorem 4, each of the functions $a_{k}(z+i)^{-k}$ $(k=1,2, \cdots, n)$ can be approximated by integral functions of order $\rho$, uniformly in the angle $\Theta$. This yields the required result.

Probably (iii) can be replaced by a milder condition (see $\$ 4.1$ ).

Application. Let $C$ be a parabola and its "interior" $R$ be defined as the part of the plane lying on its concave or convex side, respectively, and let $F(z)$ satisfy the conditions of Theorem 6 . In the first case $F(z)$ can be uniformly approximated by integral functions of any order $\rho>1 / 2$. It can be shown, however, that in the second case $F(z)$ cannot be approximated uniformly by integral functions of any finite order.

\section{§3. ON FUnCTIONS ANALYTiC AND Bounded in A HALF-PLANE}

3.1. Approximation in the mean in a half plane. In this section we take $\Theta=\pi, \rho=1$. The results will be sharper than those obtained in the general case (see \$2). We shall deal not only with functions which are bounded in the ordinary sense, but also with the class $\mathfrak{S}_{p}$. In the latter case uniform convergence will be replaced by convergence in the mean of order $p$ when $0<p<\infty$. We shall prove

THEOREM $4^{\prime}(\mathrm{a})$. In order that, uniformly in the half-plane, the function $F(z)$ can be approximated by functions $g_{\alpha}(z) \in G_{\alpha}(0<\alpha \rightarrow \infty)$ which belong to $\mathfrak{S}_{\infty}$, it is necessary and sufficient that (i) $F(z)$ belong to $\mathfrak{S}_{\infty}$ and (ii) its limit function $F(x)$ is uniformly continuous in $(-\infty, \infty)$.

TheOREM $4^{\prime}(\mathrm{b})$. Let $0<p<\infty$. In order that there should exist functions $g_{\alpha}(z) \in G_{\alpha}(0<\alpha<\infty)$ belonging to $\mathfrak{S}_{p}$ and such that, uniformly with respect to $y$,

$$
\left|F(x+i y)-g_{\alpha}(x+i y)\right|_{p} \rightarrow 0(\alpha \rightarrow \infty, 0<y<\infty),
$$

it is necessary and sufficient that $F(z)$ belong to $\mathfrak{S}_{p}$.

Without proof we state

THEOREM $4^{\prime \prime}$. Let $F(z) \in \mathfrak{S}_{p}(0<p \leqq \infty)$. Then there are functions $g_{\alpha}(z) \in G_{\alpha}$ $(0<\alpha<\infty)$ such that for any $\epsilon>0, d^{n} g_{\alpha}(z) / d z^{n} \rightarrow d^{n} F(z) / d z^{n}(n=0,1,2, \cdots$; $\alpha \rightarrow \infty)$ uniformly in the half-plane $\epsilon \leqq y<\infty$.

(11) J. L. Walsh, Interpolation and approximation by rational functions in the complex domain, Amer. Math. Soc. Colloquium Publications, vol. 20, 1935, §2.4. 
Proof of Theorem $4^{\prime}(\mathrm{a})$. The necessity of (i) follows from a known theorem, that of (ii) from Theorem $3(\mathrm{~b}) \mathrm{F}$. The sufficiency of the conditions is deduced from the Lemmas $4 \mathrm{~F}$ and 8 and from Theorem 3(b)F by means of a maximum-modulus theorem.

3.2. Proof of Theorem $4^{\prime}(\mathrm{b})$. The necessity of the condition follows from a known result. To prove its sufficiency, we take first $1 \leqq p<\infty$. The limitfunction $F(x)$ belongs to $L_{p}(-\infty, \infty)$, while $\kappa(t)$ (see $\$ 2.4$ ) belongs to $L_{1}(-\infty, \infty)$. By Lemma $4 \mathrm{~F}$, therefore, $g_{\alpha}(z)$ belongs to $G_{\alpha}$. Using Lemma 1 and the known results $\left({ }^{12}\right): F(z)$ is bounded for $y \geqq \delta(\delta>0)$, and

$$
|F(t+i \delta)-F(t)|_{p} \rightarrow 0 \quad(\delta \downarrow 0), \quad|\kappa(t-z)|_{p^{\prime}} \leqq e^{y}|\kappa(t)|_{p^{\prime}} \leqq B,
$$

where $z$ is fixed $(y>0)$ and $p^{\prime}=p(p-1)^{-1}$, we can apply the Cauchy theorem. Thus we deduce that

$$
g_{\alpha}(z)=\alpha \int_{i y-\infty}^{i y+\infty} \kappa(\alpha \tau-\alpha z) F(\tau) d \tau=\alpha \int_{-\infty}^{\infty} \kappa(\alpha t) F(t+x+i y) d t .
$$

By a well known convexity theorem, we have

$$
\left|g_{\alpha}(z)\right|^{p} \leqq|\kappa(t)|_{1}^{p-1} \int_{-\infty}^{\infty}|\kappa(t)|\left|F\left(t \alpha^{-1}+x+i y\right)\right|^{p} d t .
$$

Hence

$$
\begin{aligned}
\int_{-\infty}^{\infty}\left|g_{\alpha}(x+i y)\right|^{p} d x & \leqq|\kappa(t)|_{1}^{p-1} \int_{-\infty}^{\infty}|\kappa(t)| \int_{-\infty}^{\infty}|F(x+i y)|^{p} d x d t \\
& \leqq B_{p}^{p}|\kappa(t)|_{1}^{p},
\end{aligned}
$$

where $B_{p}$ is the upper bound of $|F(x+i y)|_{p}$ for $0<y<\infty$. Thus $g_{\alpha}(z) \in \mathfrak{E}_{p}$. Using theorem $3(\mathrm{a}) F$ and a maximum-modulus theorem due to Hille and Tamarkin $\left({ }^{13}\right)$, the proof is now completed as that of Theorem 6 .

3.3. The case $0<p<1$. To cope with the case $0<p<1$, we represent $F(z)$ in the form $\left({ }^{14}\right) F(z)=\phi(z) H(z)$ where $H(z) \in \mathscr{S}_{\infty}$, while $\Phi(z)$ belongs to $\mathfrak{S}_{p}$ and has no zero for $y>0$. Let $k$ be an integer not smaller than $p^{-1}$ and $k p=q$, so that $q \geqq 1$. The function

$$
\psi(z)=\{\phi(z)\}^{1 / k}
$$

belongs to $\mathfrak{W}_{q}$. Let $H(x), \Phi(x), \psi(x)$ be the limit-functions of $H(z), \Phi(z), \psi(z)$ respectively; then $\phi(x)=\{\psi(x)\}^{k}$. By the result of $\S 3.2$, there are functions $f_{\beta}(z) \in G_{\beta}(0<\beta<\infty)$ belonging to $\mathfrak{S}_{q}$ and such that $\left|f_{\beta}(x)-\psi(x)\right|_{q} \rightarrow 0$ as $\beta \rightarrow \infty$. Let $Q_{\beta}(z)=\left\{f_{\beta}(z)\right\}^{k}$. Then $Q_{\beta}(z) \in G_{k \beta}$ and, by Lemma $6 \mathrm{~F},\left|Q_{\beta}(x)-\Phi(x)\right|_{p} \rightarrow 0$ as $\beta \rightarrow \infty$. By Theorem 1 and Lemma 8 , there are functions $h_{\gamma}(z) \in G_{\gamma}$ $(0<\gamma<\infty)$ belonging to $\mathfrak{S}_{\infty}$ and such that, for almost all $h$ in $(-\infty, \infty)$,

(12) Hille and Tamarkin, loc. cit., Lemma 2.4 and Theorem 2.1 (iii), part 1; Lemma 2'F.

(13) Loc. cit., Theorem 2.1 (iii), part 2.

(14) Hille and Tamarkin, loc. cit., Theorem 2.2, and T. Kawata, loc. cit. 
$h_{\gamma}(x) \rightarrow H(x)$ as $\gamma \rightarrow \infty$. It is easy to see that $\left|h_{\gamma}(z)\right| \leqq A(y>0)$ where $A$ is independent of $\gamma$ (see (1.51)). Now the function $g_{\alpha}(z)=Q_{\beta}(z) h_{\gamma}(z)$ belongs to $\mathfrak{S}_{p}$ and to $G_{\alpha}$, where $\alpha=k \beta+\gamma$, and we have

$$
\begin{aligned}
\int_{-\infty}^{\infty}\left|F(x)-g_{\alpha}(x)\right|^{p} d x \leqq & \text { ess. u.b. }|H(x)|^{p} \int_{-\infty<x<\infty}^{\infty}\left|\phi(x)-Q_{\beta}(x)\right|^{p} d x \\
& +\int_{-\infty}^{\infty}\left|Q_{\beta}(x)\right|^{p}\left|H(x)-h_{\gamma}(x)\right|^{p} d x .
\end{aligned}
$$

Given $\epsilon>0$, we can fix $\beta$ so that the first term on the right is smaller than $\epsilon / 2$. Then, by the Lebesgue convergence theorem, we can fix $\gamma_{0}$ so that the second term is less than $\epsilon / 2$ for $\gamma \geqq \gamma_{0}$; for $\left|H(x)-h_{\gamma}(x)\right|^{p} \leqq\left(|H(x)|_{\infty}+A\right)^{p}$. Thus $\left|F(x)-g_{\alpha}(x)\right|_{p \rightarrow 0}$ as $\alpha \rightarrow \infty$, and applying the Hille-Tamarkin maximummodulus theorem, we arrive at (3.11).

3.4. Best approximation. In consequence of the above maximum-modulus theorem, the results of $\S 9 \mathrm{~F}$ on best approximation can be extended to the case $F(z) \in \mathfrak{S}_{p}$ (compare $\$ 2.6$ ). In the case $p=2$, in particular, the sequence of best approximation is

$$
g_{\alpha}(z)=D_{\alpha}(F ; z)=\frac{1}{\pi} \int_{-\infty}^{\infty} \frac{\sin \alpha(t-z)}{t-z} F(t) d t \quad(0<\alpha \rightarrow \infty) .
$$

\section{§4. ON FUNCTIONS WHICH ARE ANALYTIC IN AN ANGLE BUT}

\section{NOT NECESSARILY BOUNDED}

4.1. Introduction. If, uniformly in a region $R$ extending to infinity, a function $F(z)$ is approximated by rational functions, it is necessary that there exist a polynomial $f(z)$ such that $F(z)-f(z)$ tends to a finite limit uniformly for $z$ in $R$ as $|z| \rightarrow \infty$. Obviously this is a very restrictive condition.

Consider first bounded functions. In this case we can take $f(z)=0$. Functions which oscillate in the region concerned, or on its boundary, near $z=\infty$, for instance $F_{a}(z)=\exp \left(z^{a}\right)\left(1 / 2<a \leqq 1, \pi(2 a)^{-1} \leqq \arg z \leqq 3 \pi(2 a)^{-1}\right)$, cannot be approximated uniformly by rational functions, no matter how the poles are chosen. By Theorem 4, however, $F_{a}(z)$ can be approximated by integral functions of any order $\rho \geqq a(2 a-1)^{-1}$, uniformly in the domain $\pi(2 a)^{-1}$ $\leqq \arg z \leqq 3 \pi(2 a)^{-1}$.

Now consider unbounded functions. When $r$ is not an integer, the function $z^{r}(0<r<\infty)$ cannot be approximated by rational functions uniformly on any straight line, or any curve extending to infinity. For there is no polynomial $f(z)$ such that $z^{r}-f(z)$ remains bounded on this curve, not to speak of continuity (see $\$ 2.7$ ). By integral functions of finite order, however, $z^{r}$ can be approximated uniformly for $0 \leqq \arg z \leqq \Theta$ whenever $0 \leqq \Theta<2 \pi$ (Theorems $\left.11,11^{\prime}\right)$.

On regions extending to infinity, therefore, approximation by integral 
functions appears to be a stronger tool than that by rational functions.

We shall now state some theorems on the approximation by integral functions to functions which need not be bounded.

\subsection{General theorems.}

Theorem 7. Let $0<\Theta<2 \pi, \rho=\pi(2 \pi-\Theta)^{-1}$. If $F(z)$ is analytic in the angle $\Theta(0<\arg z<\Theta)$, and if there is an integral function $h(z)$ of order not greater than $\rho$ such that $F(z)-h(z)$ is bounded in the angle, and that
(a) $F(z)-h(z)$ or
(b) $F\left(z^{2}\right)-h\left(z^{2}\right)$

is uniformly continuous on the two straight lines forming the angle (a) $\Theta$ or (b) $\Theta / 2$, for $\pi \leqq \Theta<2 \pi$ or $0<\Theta<\pi$ respectively, then there are integral functions of order $\rho$ which approximate to $F(z)$ uniformly in the angle $\Theta$.

This follows immediately from Theorem 4 . For its conditions are satisfied by the function $F(z)-h(z)$.

THEOREM 7'. The function $F(z)$ can, uniformly in the upper half-plane, be approximated by functions $g_{\alpha}(z) \in G_{\alpha}(0<\alpha \rightarrow \infty)$ if, and only if, there is an integral function $h(z)$ satisfying an inequality $|h(z)|<A \exp (b|z| \rho)$ for some $\rho \leqq 1$ and such that $F(z)-h(z)$ is bounded in the half-plane and uniformly continuous on the real axis.

The sufficiency of the conditions is obvious; so is their necessity, taking, for instance, $h(z)=g_{1}(z)$. A similar result holds for the approximation in the mean of order $p(0<p<\infty)$.

THEOREM 8. Let, uniformly in the angle $\Theta(0<\Theta<2 \pi), F(z)$ be approximated by integral functions of finite types of the order $\rho=\pi(2 \pi-\Theta)^{-1}$, and let the limit inferior of the types be a finite number $\beta$. Then $F(z)$ is an integral function, satisfying an inequality $|F(z)|<A_{\epsilon} \exp \{(\beta+\epsilon)|z| \rho\}$ for any $\epsilon>0$.

The proof is based upon Lemma 3, observing that the difference of any two of the approximating functions is an integral function of finite type of the order $\rho$, bounded in the angle $\Theta$, and using an argument presented in $\S 8 \mathrm{~F}$.

4.3. A Lipschitz condition. When we try to apply Theorems 7 or $7^{\prime}$ to a given function $F(z)$ which is not bounded then a particular difficulty arises. There is no general criterion for the existence of a function $h(z)$ as required in the theorems.

We shall deal with special cases, using another method. We start with a Lipschitz condition.

Theorem 9. Let $1 \leqq \rho=\pi(2 \pi-\Theta)^{-1}$ and $0<r \leqq 1$; and let $k$ be a positive integer. If

(i) $F(z)$ is analytic for $|\arg z-\pi / 2|<\Theta / 2$ and, uniformly for $|\arg z-\pi / 2|$ $<\Theta / 2, F(z)=O\left(|z|^{k}\right)$ as $|z| \rightarrow \infty$, and 
(ii) $|F(z+t)-F(z)| \leqq A|t|^{r}$ uniformly for $-\infty<t<\infty, 0<y<\infty$, and

(iii) $|F(z+\tau)-F(z)| \leqq A|\tau| r$ uniformly with respect to $u, t$ and $\phi$, where $z=u e^{i \phi}, \tau=t e^{i \phi},-\infty<u<\infty,-\infty<t<\infty,|\phi|<(\Theta-\pi) / 2$, then there is $a$ set of integral functions $g_{\alpha}(z)$ such that $\left|g_{\alpha}(z)\right|<A_{\alpha, \epsilon} \exp (|(\alpha+\epsilon) z| \rho)$ for any $\epsilon>0$ and that, uniformly for $|\arg z-\pi / 2|<\Theta / 2, g_{\alpha}(z) \rightarrow F(z)$ as $\alpha \rightarrow \infty$.

Evidently (iii) is to be dropped when $\rho=1, \Theta=\pi$. By means of a conformal transformation we deduce that the limit-function of $F(z)$ exists at any finite point of the two lines arg $z= \pm(\Theta-\pi) / 2$ and that, therefore, (iii) holds on these two lines. We set

$$
g_{\alpha}(z)=\alpha \int_{-\infty}^{\infty} \kappa\{\alpha(t-z)\} F(t) d t
$$

where $\kappa(z)$ is defined as in $\$ 2.4$ and $t^{k+2} \kappa(t)$ is bounded in $(-\infty, \infty)$. By Lemma $7, g_{\alpha}(z)$ is an integral function satisfying (2.23). Fixing $z$ in the upper half-plane and observing that, uniformly for $0<\Im(\tau) \leqq y, \kappa\{\alpha(\tau-z)\} \tau^{k} \rightarrow 0$ as $|\tau| \rightarrow \infty$, we can apply the Cauchy theorem to move the path of integration. Thus we deduce that

$$
g_{\alpha}(z)=\alpha \int_{-\infty}^{\infty} \kappa(\alpha t) F(t+z) d t .
$$

Hence, using (ii), we have

$$
\begin{aligned}
\left|g_{\alpha}(z)-F(z)\right| & \leqq \int_{-\infty}^{\infty}|\kappa(t)||F(t / \alpha+z)-F(z)| d t \\
& \leqq A \alpha^{-r} \int_{-\infty}^{\infty}\left|\kappa(t) t^{r}\right| d t
\end{aligned}
$$

The term on the right tends to zero as $\alpha \rightarrow \infty$, uniformly for $0<y<\infty$.

Observing that $t^{k} \kappa\left(\alpha t e^{i \psi}-\alpha u e^{i \phi}\right) \rightarrow 0$ as $t \rightarrow \pm \infty(u, \phi$ fixed, $|\phi|<(\Theta-\pi) / 2)$, uniformly for $0 \leqq \psi \leqq \phi$ or $\phi \leqq \psi \leqq 0$, we can turn the path of integration in (4.31); hence

$$
\mathrm{g}_{\alpha}(z)=\alpha e^{i \phi} \int_{-\infty}^{\infty} \kappa\left\{\alpha e^{i \phi}(t-u)\right\} F\left(t e^{i \phi}\right) d t \quad\left(z=u e^{i \phi}\right) .
$$

Using (iii) it can now be shown that $g_{\alpha}(z) \rightarrow F(z)$ as $\alpha \rightarrow \infty$ (see 4.33) uniformly for $|\phi|<(\Theta-\pi) / 2$. This completes the proof.

\subsection{A Taylor condition.}

TheOREM 10. Let, uniformly for $|\arg z-\pi / 2|<\Theta / 2$, the functions $F_{j}(z)$ $(j=1,2, \cdots, n)$ be approximated by integral functions of finite types of the order $\rho=\pi(2 \pi-\Theta)^{-1}(\rho \geqq 1)$; let $F(z)$ satisfy. condition (i) of Theorem 9, and let $0<r_{1}<r_{2}<\cdots<r_{n}<\infty$ and 


$$
F(z+\tau)=F(z)+\sum_{j=1}^{n} \tau^{r j} F_{j}(z)+R(z ; \tau)
$$

where, uniformly for $0<y<\infty,-\infty<\tau<\infty$ (arg $\tau=0$ or $\pi$ ), and uniformly for $-\infty<u<\infty,-\infty<v<\infty,(\pi-\Theta) / 2<\phi<(\Theta-\pi) / 2\left(z=u e^{i \phi}, \tau=v e^{i \phi}\right)$,

$$
|R(z ; \tau)| \leqq A_{0}|\tau|^{c} \quad(0<c<\infty) .
$$

Then, uniformly for $|\arg z-\pi / 2|<\Theta / 2, F(z)$ can be approximated by integral functions of finite types of the order $\rho$.

Let $l>\max \left(k / 2+1, r_{n} / 2+1, c / 2+1\right)$ (see $\left.\$ 1.4(1.43)\right)$, and let $g_{\alpha}(z)$ be defined by (4.31). Then we have

$$
g_{\alpha}(z)-F(z)=\sum_{j=1}^{n} \alpha^{-r_{j}} F_{j}(z) \int_{-\infty}^{\infty} \kappa(t) t^{r_{j}} d t+\alpha \int_{-\infty}^{\infty} \kappa(\alpha t) R(z ; t) d t
$$

The last term is majorized by $A_{0} \alpha^{-c}$, as we see by estimating the integral first for $y>0$ and then for $|\arg z|<(\Theta-\pi) / 2$ and for $|\arg z-\pi|<(\Theta-\pi) / 2$.

From the hypotheses we deduce that the integrals $\int_{-\infty}^{\infty} k|t| t^{r i} d t$ are finite constants $c_{j}$, and that there are integral functions $g_{\alpha, j}(z)$ of the type $\alpha^{\rho}$ of the order $\rho$ such that $F_{j}(z)-g_{\alpha, j}(z)$ is bounded uniformly with respect to $z$ for $|\arg z-\pi / 2|<\Theta / 2$ and to $\alpha$ for $\alpha_{0}<\alpha<\infty\left(\alpha_{0}>0\right)$, with upper bound $A_{j}$, say. Hence, taking $\tilde{g}_{\alpha}(z)=g_{\alpha}(z)-\sum c_{j} \alpha^{-r i} g_{\alpha, j}(z)$, we have

$$
\left|F(z)-\tilde{g}_{\alpha}(z)\right| \leqq \sum_{j=1}^{n} \alpha^{-r_{i}} A_{i}\left|c_{j}\right|+\alpha^{-c} A_{0} \rightarrow 0 \quad \text { as } \quad \alpha \rightarrow \infty
$$

uniformly for $|\arg z-\pi / 2|<\Theta / 2$. Thus we have proved the theorem.

4.5. An extension of the binomial theorem. The equality which will now be proved is treated in more detail than needed for our purpose, since it can be regarded as an extension of the binomial theorem.

Lemma 9. Let $0 \leqq \arg z \leqq \pi,-\infty<t<\infty, 0 \leqq$ arg $(z+t) \leqq \pi$; let $n$ be an integer and $0 \leqq n<r \leqq n+1$. Then, uniformly with respect to $z$,

$$
\left|(z+t)^{r}-\sum_{j=0}^{n} C_{r, j} t^{j} z^{r-j}\right| \leqq A_{r}|t| r,
$$

where the best possible value of $A_{r}$ is unity for $0<r-n \leqq 1 / 2$ and for $r=n+1$, while it is not greater than $2^{n+1-r} \sin \pi(r-n) / 2$ for $1 / 2<r-n<1$.

LEMMA $9 '$. The result holds, uniformly with respect to $\phi, t$ and $z$, if $\arg z$, arg $t$, arg $(z+t)$ take the values $\phi$ and $\phi+\pi$ only ( $\phi$ real).

This follows from (4.51), taking $z$ real and turning the real axis by the angle $-\phi$.

Proof of Lemma 9. Let $0<r<1$. The function $h(z)=(z+t)^{r}-z^{r}$ belongs 
to $\mathfrak{W}_{\infty}$. Hence, by a maximum-modulus theorem, we have

$$
\begin{aligned}
\left|(z+t)^{r}-z^{r}\right| \leqq|h(x)|_{\infty} & =\underset{-\infty<x<\infty}{\text { u.b. }}\left|(x+t)^{r}-x^{r}\right| \\
& \leqq \underset{-\infty<x_{1}<\infty,-\infty<x<\infty}{\text { u.b. }}\left|x_{1}^{r}-x^{r}\right| \quad\left(x_{1}=x+t\right) .
\end{aligned}
$$

When $x$ and $x_{1}$ are both either positive or negative, then we have

$$
\left|x_{1}^{r}-x^{r}\right| \leqq\left|x_{1}-x\right|^{r}=|t|^{r} \text {. }
$$

Let $x<0, x_{1}>0$. Then $\left|x_{1}^{r}-x^{r}\right| \leqq x_{1}^{r}+|x|^{r} \leqq 2^{1-r}\left(x_{1}+|x|\right)^{r}=2^{1-r} t^{r}$. Hence certainly $A_{r} \leqq 2^{1-r}$. Putting $x_{1} x^{-1}=-s$, we have

$$
\left|\frac{x_{1}^{r}-x^{r}}{\left(x_{1}-x\right)^{r}}\right|=\frac{\left|s^{r}-e^{i \pi r}\right|}{(s+1)^{r}}=V_{r}(s) \quad(0<s<\infty) .
$$

Taking $W_{r}(s)=s^{-r}(s+1)^{2 r}\left\{1-\left(V_{r}(s)\right)^{2}\right\}$, we have $W_{r}(1)=2^{2 r}+2 \cos \pi r-2$. Now we need some inequalities.

$$
\begin{aligned}
& \begin{aligned}
& \cos \pi r>1-2 r \\
&<1-2 r
\end{aligned} \text { for } \begin{aligned}
0 & <r<1 / 2 \\
1 / 2 & <r<1
\end{aligned} \\
& 4^{r}-4 r>0 \quad \text { for } \quad 0<r<1 / 2 \text {, } \\
& <0 \quad 1 / 2<r<1 \text {. } \\
& \left(1-s^{a}\right)(1-s)^{-1} \leqq(1+s)^{a-1} \quad(0<a \leqq 1 ; 0<s<\infty) .
\end{aligned}
$$

Possibly (4.54) is known, while (4.52) and (4.53) are obvious.

First we suppose that $0<r \leqq 1 / 2$. We have

$$
\frac{d W_{r}(s)}{d s}=\frac{r(s-1)}{s^{r+1}}\left\{(s+1)^{2 r-1}-\frac{1-s^{2 r}}{1-s}\right\} .
$$

By (4.54), the term in braces is not negative. Hence $W_{r}(1)=2^{2 r}+2 \cos \pi r-2$ is the minimum value of $W_{r}(s)$ in $(0, \infty)$. By (4.52) and (4.53), we have $W_{r}(1) \geqq 4^{r}-4 r \geqq 0$. Hence $V_{r}(s) \leqq 1$, and so $A_{r} \leqq 1$. Taking $x_{1} \rightarrow \infty$ we see that $A_{r}=1$.

If $1 / 2<r<1$, then $W_{r}(1)<4^{r}-4 r<0$, therefore $V_{r}(1)>1$, and so $A_{r}>1$. Since $s^{r}(s+1)^{-2 r} \leqq 4^{-r}$, we have $\left\{V_{r}(s)\right\}^{2}-1 \leqq 4^{-r}\left|W_{r}(1)\right|$, and so $A_{r}=V_{r}(1)$ $=2^{1-r} \sin \pi r / 2$. Hence the lemma is true for $0<r<1$, even in a slightly sharper form than stated above. For $n<r \leqq n+1$, we deduce that the left side of (4.51) is equal to

$$
r(r-1) \cdots(r-n+1)\left|\int_{0}^{t} d t_{1} \int_{0}^{t_{1}} d t_{2} \cdots \int_{0}^{t_{n-1}}\left\{\left(z+t_{n}\right)^{r-n}-z^{r-n}\right\} d t_{n}\right|
$$


Since $0<r-n \leqq 1$, the modulus of the term in braces is not greater than $A_{r-n}\left|t_{n}\right|^{r-n}$. Thus the required result follows at once.

4.6. Application to the function $z^{r}$. We suppose that $-\pi / 2<\arg z<3 \pi / 2$.

TheOREM 11. When $0<\Theta<\pi$ and $\rho=\pi(2 \pi-\Theta)^{-1}$ then, uniformly for $|\arg z-\pi / 2|<\Theta / 2$, the function $z^{r}(0<r<\infty)$ can be approximated by integral functions of finite types of the order $\rho$, the types tending to infinity.

First we take $\pi \leqq \Theta<2 \pi$. For $0<r \leqq 1$, the theorem is an immediate consequence of (4.51), with $n=0$, and of Theorem 9. Suppose that it holds for $0<r \leqq n$, where $n$ is a positive integer. Then, by (4.51) and Theorem 10 , it holds for $n<r \leqq n+1$, which completes the proof.

The case $0<\Theta<\pi$ is treated in a similar way as the corresponding one in $\$ 2.5$, but using some result corresponding to Theorem 10 and the first part of

Lemma $9^{\prime \prime}$. Let $0 \leqq n<2 r \leqq n+1,-\infty<t, u<\infty$. Then, for even or odd values of $n$, respectively,

$$
\left|\left\{(u+t)^{2}\right\} r-\sum_{j=0}^{n} C_{2 r, j}(t \operatorname{sgn} u)^{i}\left(u^{2}\right)^{r-j / 2}\right| \leqq\left(t^{2}\right)^{r} \text { or } 2^{n+1-2 r}\left(t^{2}\right)^{r},
$$

and

$$
\left|\left\{(u+t)^{2}\right\} r \operatorname{sgn}(u+t)-\sum_{j=0}^{n} C_{2 r, j} t^{j}(\operatorname{sgn} u)^{j+1}\left(u^{2}\right)^{r-j / 2}\right| \leqq 2^{n+1-2 r}\left(t^{2}\right)^{r} \text { or }\left(t^{2}\right)^{r} .
$$

We observe that, with a suitable choice of $\kappa(t), \int_{-\infty}^{\infty} \kappa(t) t^{j} d t=0$ for $j=1,3,5, \cdots(j \leqq n)$.

It can be shown that the theorem holds in the limiting case $\Theta=0$ :

THEOREM 11'. The function $x^{r}$ can, uniformly in $(0, \infty)$, be approximated by integral functions of finite types of the order $1 / 2$.

\section{§5. THE STRIP}

5.1. Approximation in the mean in a strip. Let $a$ and $b$ be finite real numbers $(a<b)$ and $0<p \leqq \infty$. Let $S$ be the region $-\infty<x<\infty, a<y<b$, and let $S_{p}(a, b)$ be the set of functions which are analytic in $S$ and satisfy the condition

$$
|F(x+i y)|_{p} \leqq A_{p} \quad(a<y<b),
$$

where $A_{p}$ does not depend on $y$. We shall prove

ThEOREM 12. (a) Let $0<p<\infty$ and $F(z) \in S_{p}(a, b)$. Then there are functions $g_{\alpha}(z) \in G_{\alpha}(0<\alpha<\infty)$ such that, uniformly for $a<y<b, \mid F(x+i y)$ $-\left.g_{\alpha}(x+i y)\right|_{p} \rightarrow 0$ as $\alpha \rightarrow \infty$.

(b) Let $F(z) \in S_{\infty}(a, b)$, that is to say, let $F(z)$ be analytic and bounded in $S$, 
and let $F(x+i a)$ and $F(x+i b)$ be its limit-functions. Then $F(z)$ can be approximated, uniformly in $S$, by functions $g_{\alpha}(z) \in G_{\alpha}(0<\alpha<\infty)$ if and only if the limit-functions $F(x+i a)$ and $F(x+i b)$ are uniformly continuous in $(-\infty, \infty)$. We note that the $g_{\alpha}(z)$ can be replaced by integral functions of any higher order, but not of any smaller one.

The proof is based upon a theorem which corresponds to results of HilleTamarkin on the class $\mathfrak{S}_{p}$. Its proof will be given in the appendix to the present paper.

\subsection{The factorization theorem.}

THEOREM 13. Let $0<p \leqq \infty$ and $F(z) \in S_{p}(a, b)$.

(a) Then $F(z)$ is represented in the form $F(z)=G(z) H(z)$ where $G(z)$ has no zeros in the interior of the strip and belongs to $S_{p}(a, b)$, while $H(z)$ belongs to $S_{\infty}(a, b)$ and $|H(x+i a)|=|H(x+i b)|=1$ for almost all $x$ in $(-\infty, \infty)$.

(b) For almost all $t$ in $(-\infty, \infty), F(z)$ tends to limit-functions $F(t+i a)$ or $F(t+i b)$, respectively, as $z$ tends to $t+i a$ or $t+i b$ from the interior of $S$ along any path not touching the lines bounding $S$.

(c) When $0<p<\infty$ then

$$
\left|F(x+i y)-\begin{array}{l}
F(x+i a) \\
F(x+i b)
\end{array}\right|_{p} \rightarrow 0 \quad \text { as } \quad \begin{aligned}
& y \downarrow a \\
& y \uparrow b .
\end{aligned}
$$

(c') When $p=\infty$ then $|F(x+i y)|_{\infty} \rightarrow|F(x+i a)|_{\infty}$ as $y \downarrow$ a and $|F(x+i y)|_{\infty}$ $\rightarrow|F(x+i b)|_{\infty}$ as $y \uparrow b$, while (5.21) holds for $p=\infty$ if and only if the limitfunctions $F(x+i a)$ and $F(x+i b)$ are uniformly continuous in $(-\infty, \infty)$. When this condition is satisfied, or when the limit-functions are merely continuous in $(-\infty, \infty)$, then $F(z)$ is uniformly continuous or is merely continuous, respectively, for $-\infty<x<\infty, a \leqq y \leqq b$.

(d) When $0<p \leqq \infty$ then, for $a<y<b$,

$$
|F(x+i y)|_{p} \leqq|F(x+i a)|_{p}^{(b-y) /(b-a)}|F(x+i b)|_{p}^{(y-a) /(b-a)}
$$

(e) When $1 \leqq p<\infty$, then, for $a<y<b, F(z)$ is represented by its proper Cauchy integral

$$
F(z)=\frac{1}{2 \pi i} \int_{-\infty}^{\infty} \frac{F(t+i a) d t}{t+i a-z}-\frac{1}{2 \pi i} \int_{-\infty}^{\infty} \frac{F(t+i b) d t}{t+i b-z} .
$$

(f) When $1 \leqq p \leqq \infty$ then, for $a<y<b, F(z)$ is represented by its proper Poisson integral

$$
\begin{aligned}
F(z)= & \frac{1}{\pi} \int_{-\infty}^{\infty} \frac{(y-a) F(t+i a) d t}{(t-x)^{2}+(y-a)^{2}} \\
& -\frac{1}{\pi} \int_{-\infty}^{\infty} \frac{(y-a) F(t+i b) d t}{(t-x+i b-i a)^{2}+(y-a)^{2}}
\end{aligned}
$$


and by the corresponding formula resulting by interchanging $a$ and $b$, and the signs of the integrals.

\subsection{Proof of Theorem 12. We need}

Lemma 10. Let $0<\alpha<\infty$, let $1 \leqq p \leqq \infty$ and $F(z) \in S_{p}(a, b)$. Let $\kappa(z) \in G_{1}$ and $\kappa(x) \in L_{1}(-\infty, \infty)$. Then the function

$$
g_{\alpha}(z)=\alpha \int_{-\infty+i a}^{\infty+i a} k(\alpha \tau-\alpha z) F(\tau) d \tau
$$

belongs to $S_{p}(a, b)$.

The proof is similar to that of Theorem $4^{\prime}(a, b)$, using Lemma $12(\$ 7.1)$ and Theorem 13(c).

To prove the theorem for $1 \leqq p \leqq \infty$, we suppose that $\kappa(z)$ satisfies the conditions of the lemma and that $\int_{-\infty}^{\infty} \kappa(t) d t=1$. By Theorems 13(c) and $\left(c^{\prime}\right)$, we have

$$
\begin{aligned}
& g_{\alpha}(x+i a) \\
& g_{\alpha}(x+i b)
\end{aligned}=\alpha \int_{-\infty}^{\infty} \kappa(\alpha t) d t{ }_{F(t+x+i b)}^{F(t+x+i a)} \quad(-\infty<x<\infty) .
$$

Using a result due to $\mathrm{E}$. Hille (Lemma $7 \mathrm{~F}$ ), we deduce that, for $1 \leqq p \leqq \infty$,

$$
\left|g_{\alpha}(x+i a)-F(x+i a)\right|_{p} \rightarrow 0, \quad\left|g_{\alpha}(x+i b)-F(x+i b)\right|_{p} \rightarrow 0,
$$

as $\alpha \rightarrow \infty$. Now we apply Theorem $13(\mathrm{~d})$ to the function $g_{\alpha}(z)-F(z)$, and so, uniformly for $a<y<b, g_{\alpha}(z) \rightarrow F(z)$ as $\alpha \rightarrow \infty$. The necessity of the uniform continuity of the limit-functions for $p=\infty$ is deduced as in Theorem $3(\mathrm{~b}) \mathrm{F}$. This completes the proof for $1 \leqq p \leqq \infty$. For $0<p<1$, the proof follows lines of $\$ 3.2$, but using the Theorems $13(\mathrm{a})$ and $13(\mathrm{~d})$.

\section{§6. SOME APPLICATIONS}

6.1. The Weierstrass approximation theorem. Let $f(t)$ be continuous for $0 \leqq t \leqq 1$. Given $\epsilon>0$, we take $F(t)=f(0)$ or $f(t)$ or $f(1)$ for $t<0$ or $0 \leqq t \leqq 1$ or $t>1$, respectively; by Theorem $3(\mathrm{~b}) \mathrm{F}$, we can find an integral function $g(z)$ of exponential type such that $|F(t)-g(t)|_{\infty} \leqq \epsilon / 2$. Expanding $g(z)$ into a power series, we can find a polynomial $T(z)$ such that $|g(t)-T(t)| \leqq \epsilon / 2$ for $0 \leqq t \leqq 1$. Hence $|f(t)-T(t)| \leqq \epsilon$ in $(0,1)$.

6.2. A property of the Dirichlet singular integral. Let $F(z) \in G_{\alpha}(0<\alpha<\infty)$ and $F(t) \in L_{p}(-\infty, \infty)(0<p<\infty)$. Then $F(z)=D_{\beta}(F ; z)$ (see (3.41)) whenever $\infty>\beta \geqq \alpha$.

This result is known $\left({ }^{15}\right)$. Here we give another proof. Let $0<p \leqq 2$. By Lemma $2^{\prime}(\mathrm{a}) \mathrm{F}$, the function $F(x)$ belongs to $L_{2}(-\infty, \infty)$. Among all elements of $G_{\beta}$, the function $\dot{D}_{\beta}(F ; z)$ is that of best approximation to $F(x)$ in $L_{2}(-\infty, \infty)$ (Theorem $\left.5^{\prime} F\right)$. But certainly $F(z) \in G_{\beta}$ and $|F(x)-F(x)|_{2}=0$,

(15) H. Kober, Quart. J. Math. Oxford Ser. vol. 11 (1940) pp. 66-80, Theorem 6. 
which yields the required result for $0<p \leqq 2$. When $2<p<\infty$, we apply the preceding result to the function $(\epsilon z)^{-1} \sin \epsilon z F(z)$ and take $\epsilon \rightarrow 0$; thus we prove the assertion for $\beta>\alpha$ and, by continuity, for $\beta=\alpha$.

6.3. Approximation by rational functions. We use the notation of $\S 5$.

TheOREM 14. Let $0<p<\infty$ and $F(z) \in S_{p}(a, b)$, and let $z_{1}=u_{1}+i v_{1}$, $z_{2}=u_{2}+i v_{2}$ be any two fixed points outside the strip $S$, lying in the lower or upper half-plane, respectively. Then there are rational functions $f_{n}(z)(n=1,2, \cdots)$, with their only poles at $z_{1}$ and $z_{2}$ and such that, uniformly for $a<y<b$,

$$
\left|f_{n}(x+i y)-F(x+i y)\right|_{p} \rightarrow 0 \text { as } n \rightarrow \infty .
$$

It is known (16) that the result is true for $p=\infty$ under the additional condition that the two limit-functions are continuous in $(-\infty, \infty)$, and that the four limits, as $x \rightarrow \pm \infty$ in $F(x+i a)$ and $F(x+i b)$, exist and are finite and equal. To deal with the case $0<p<\infty$, we need

LEMMA 11. Let $F(z)$ be analytic and bounded for $y<0$ and $F(x)$ be its limitfunction as $y \rightarrow 0$, and let $z_{0}$ be any fixed point in the upper half-plane. Then there are rational functions $r_{n}(z)(n=1,2, \cdots)$ with the following properties:

(I) They have no singularity except at $z=z_{0}$.

(II) $r_{n}(x) \rightarrow F(x)$ at almost all $x$ in $(-\infty, \infty)$, as $n \rightarrow \infty$.

(III) $r_{n}(z) \rightarrow F(z)$, as $n \rightarrow \infty$, uniformly in any finite domain of the halfplane $y<0$.

(IV) $\left|r_{n}(z)\right| \leqq|F(x)|_{\infty}$ for $y \leqq 0$.

If $F(x)$ is continuous in $(-\infty, \infty)$, then (II) holds uniformly in any finite interval.

The proof of the lemma is given in the appendix to the present paper, but that of its last assertion is omitted.

By Theorem 12(a), there are functions $g_{\alpha}(z) \in G_{\alpha}(0<\alpha<\infty)$ such that, uniformly for $a<y<b,\left|F(x+i y)-g_{\alpha}(x+i y)\right|_{p} \rightarrow 0$ as $\alpha \rightarrow \infty$. Hence, uniformly for $a<y<b$,

$$
\left|F(x+i y)-e^{-i \alpha(x+i y)} H_{\alpha}(x+i y-i a)\right|_{p} \rightarrow 0 \text { as } \alpha \rightarrow \infty,
$$

where $H_{\alpha}(z-i a)=e^{i \alpha z} g_{\alpha}(z)$. By Lemma $2^{\prime}(\mathrm{b}) \mathrm{F}$, with $P(z)=1, H_{\alpha}(z)$ belongs to $\mathfrak{S}_{p}$. Now we fix $\alpha$. By a known result $\left({ }^{17}\right)$, there are rational functions $R_{n}(z)(n=1,2, \cdots)$, with their only singularity at the point $z^{\prime}=z_{1}-i a$, and such that $\left|H_{\alpha}(x+i y)-R_{n}(x+i y)\right|_{p} \rightarrow 0$ as $n \rightarrow \infty$ uniformly for $y>0$. Hence

$$
\left|e^{-i \alpha(x+i y)}\left\{H_{\alpha}(x+i y-i a)-R_{n}(x+i y-i a)\right\}\right|_{p} \rightarrow 0 \text { as } n \rightarrow \infty
$$

uniformly in the strip $a<y<b$.

The function exp $\{-i \alpha(z+i b)\}$ satisfies the condition of Lemma 11 . Hence there are rational functions, $r_{m}(z)(m=1,2, \cdots)$ say, with a single

(16) J. L. Walsh, loc. cit.

(17) H. Kober, Bull. Amer. Math. Soc. vol. 49 (1943) pp. 437-443, Theorem 2(b). 
singularity at $z_{2}-i b$ and such that $\left|r_{m}(z)\right| \leqq e^{\alpha b}$ for $y \leqq 0$ and that $r_{m}(z-i b)$ $\rightarrow \exp (-i \alpha z)$ as $m \rightarrow \infty$ at almost all (even at all) points of the two lines $y=a, y=b$. Fixing $n$ and applying the Lebesgue convergence theorem, we have

$$
\left|\left\{e^{-i \alpha(x+i y)}-r_{m}(x+i y-i b)\right\} R_{n}(x+i y-i a)\right|_{p} \rightarrow 0 \text { as } m \rightarrow \infty
$$

for $y=a$ and $y=b$ and, by Theorem 13(d), therefore, uniformly for $a<y<b$. Combining (6.32), (6.33) and (6.34), we can construct a sequence $f_{n}(z)$ $(n=1,2, \cdots)$ of the kind required in the theorem. This completes the proof.

For $1<p<\infty$ the result can also be deduced from (5.23). Denoting the terms on the right of $(5.23)$ by $F_{1}(z)$ and $F_{2}(z)$, it can be shown that both $F_{1}(z+i a)$ and $F_{2}(i b-z)$ belong to $\mathfrak{S}_{p}$. The proof is completed by employing a known result $\left({ }^{17}\right)$ on the approximation to elements of $\mathfrak{S}_{p}$ by rational functions. This method, however, fails for $p=1$ and $0<p<1$.

\section{§7. APPENDIX}

7.1. Estimates in the strip. For $p=2$, the assertions (b), (c), (e) of Theorem 13 have been proved by E. C. Titchmarsh $\left({ }^{18}\right)$. For $p=\infty$, (b) is well known, so is probably (d). The proof follows the lines of a classical proof due to F. Riesz $\left({ }^{19}\right)$, using a result due to G. H. Hardy, A. E. Ingham and G. Pólya, and the Titchmarsh theorem. Without loss of generality, we take $b=-a=\pi / 2$. We need some lemmas.

LemMA 12. Let $0<p<\infty$ and $F(z) \in S_{p}(-\pi / 2, \pi / 2)$, and let $0<\delta<\pi / 2$. Then, for $|y| \leqq \pi / 2-\delta, F(z)$ tends uniformly to zero as $|z| \rightarrow \infty$ and $|F(z)|$ $\leqq A_{p} \delta^{-2 / p}$, where $A_{p}$ is defined by (5.11).

Using results due to Hardy and an argument due to Plancherel and Pólya $\left({ }^{20}\right)$, we have

$$
\begin{aligned}
|F(z)|^{p} & \leqq \frac{1}{\pi \delta^{2}} \int_{-\delta}^{\delta} \int_{-\delta}^{\delta}|F(z+s+i t)|^{p} d s d t=\frac{1}{\pi \delta^{2}} \int_{y-\delta}^{y+\delta} d t \int_{x-\delta}^{x+\delta}|F(s+i t)|^{p} d s \\
& \leqq \frac{1}{\pi \delta^{2}} \int_{-\pi / 2}^{\pi / 2} d t \int_{x-\delta}^{x+\delta}|F(s+i t)|^{p} d s=I .
\end{aligned}
$$

By (5.11), the inner integral is uniformly bounded in $S$ and tends, for any $t$ in $(-\pi / 2, \pi / 2)$, to zero as $x \rightarrow \pm \infty$. Since $I$ is independent of $y$, we arrive at the required result by means of the Lebesgue theorem.

Using the lemma and applying the Cauchy integral theorem, we have

(18) Introduction to the theory of Fourier integrals, Oxford, 1937, Theorem 97. The proof can, however, be based entirely on the Riesz theory for $0<p<\infty$.

(19) F. Riesz, Math. Zeit. vol. 18 (1923) pp. 87-95.

(20) Comment. Math. Helv. vol. 10 (1937/1938) pp. 110-163, inequality (73). 
LEMma $12^{\prime}$. Let $0<p \leqq \infty, 0<\delta<\pi / 2$ and $F(z) \in S_{p}(-\pi / 2, \pi / 2)$; then $d^{n} F(z) / d z^{n}(n=0,1,2, \cdots)$ is bounded for $|x|<\infty,|y| \leqq \pi / 2-\delta$.

7.2. Proof of Theorem 13(a). The conformal transformation

$$
w=\left(e^{z}-1\right) /\left(e^{z}+1\right)=\tanh (z / 2)
$$

maps the interior of the unit circle $\Gamma$ of the w-plane on the strip $S$ in the $z$-plane $\left({ }^{21}\right)$. Let $F(z)=f(w)$, then $f(w)$ is regular in $\Gamma$. Let $\alpha_{1}, \alpha_{2}, \cdots$ be the zeros of $f(w)$ in $\Gamma$, in order of increasing $\left|\alpha_{k}\right|$, multiple zeros being written multiply. Let $\alpha_{k}=\tanh \left(\beta_{k} / 2\right),\left|\Im \beta_{k}\right|<\pi / 2$, then the $\beta_{k}$ 's are the zeros of $F(z)$ in $S$. First we suppose that $F(0)=f(0)$ is different from zero. We set

$$
\begin{array}{ll}
H_{n}(z)=\prod_{k=1}^{n} \frac{\sinh \left(\beta_{k}-z\right) / 2}{\cosh \left(\bar{\beta}_{k}-z\right) / 2}\left(\frac{\sinh \bar{\beta}_{k}}{\sinh \beta_{k}}\right)^{1 / 2}, & \\
h_{n}(w)=\prod_{k=1}^{n} \frac{\left|\alpha_{k}\right|}{\alpha_{k}} \frac{\alpha_{k}-w}{1-w \bar{\alpha}_{k}} \quad(n=1,2, \cdots),
\end{array}
$$

where the square roots are defined so that the factors of the product in (7.22) are positive at $z=0$. Then $H_{n}(z)=h_{n}(w)$, and the function $H_{n}(z)$ has the following properties:

$$
\begin{aligned}
& \left|H_{n}(z)\right| \rightarrow 1 \text { as } z \rightarrow x \pm i \pi / 2 . \\
& \left|H_{n}(z)\right| \rightarrow 1, \text { as }|z| \rightarrow \infty, \text { uniformly for }|y|<\pi / 2 .
\end{aligned}
$$

Any rectangle $|x| \leqq A,|y| \leqq B$, where $B<\pi / 2$, is mapped on a part of the circle $|w| \leqq R$, where $R<1$. For

$$
\begin{aligned}
|w|^{2} & =(\cosh x-\cos y)(\cosh x+\cos y)^{-1} \\
& \leqq 1-\cos y(\cosh x)^{-1} \leqq 1-\cos B / \cosh A .
\end{aligned}
$$

If, therefore, we can show that, uniformly in any circle $|w| \leqq R<1, h_{n}(w)$ $(n \rightarrow \infty)$ converges to a function $h(w)$ which is analytic in $\Gamma$, then $H_{n}(z)(n \rightarrow \infty)$ converges, uniformly in any rectangle $|x| \leqq A,|y| \leqq B<\pi / 2$, to a function $H(z)$ which is analytic in $S$. It is well known that the convergence of the infinite product $\prod\left|\alpha_{k}\right|$ is a necessary and sufficient condition for the above property of $h_{n}(w)$. Now we have

$$
\prod_{k=1}^{n}\left|\alpha_{k}\right|=h_{n}(0)=H_{n}(0) \text {. }
$$

Therefore we have only to show that $H_{n}(0) \geqq B_{0}$, where $B_{0}$ is independent of $n$. Let $F(z)=G_{n}(z) H_{n}(z)$. Given $\epsilon>0$, by (7.24) and (7.25) we have $\left|H_{n}(z)\right| \geqq 1-\epsilon$ for $\pi / 2-\delta \leqq|y| \leqq \pi / 2$, where $\delta$ depends on $\epsilon$ and $n$. Hence, for $0<p \leqq \infty$, we have

(21) Compare Hardy, Ingham, P6lya, loc. cit., Theorem 1. 


$$
\begin{aligned}
\left|G_{n}(x+i y)\right|_{p} \leqq(1-\epsilon)^{-1}|F(x+i y)|_{p} \leqq & (1-\epsilon)^{-1} A_{p} \\
& (\pi / 2-\delta \leqq|y|<\pi / 2)
\end{aligned}
$$

where $A_{p}$ is defined by (5.11). In consequence of Lemma 12, the function $G_{n}(z)=F(z) / H_{n}(z)$ is bounded for $|y| \leqq \pi / 2-\delta$. Applying the Hardy-InghamPólya theorem( $\left.{ }^{22}\right)$ to $G_{n}(z)$ in the closed strip $|y| \leqq \pi / 2-\delta$ and using (7.27), with $y= \pm(\pi / 2-\delta)$, we deduce that $\left|G_{n}(x+i y)\right|_{p} \leqq A_{p}(1-\epsilon)^{-1}$ for $|y| \leqq \pi / 2-\delta$. Hence, for $|y|<\pi / 2$, we have

$$
\left|G_{n}(x+i y)\right|_{p} \leqq A_{p}(1-\epsilon)^{-1} \rightarrow A_{p} \quad \text { as } \epsilon \rightarrow 0 .
$$

Using Lemma 12 , we have $\left|G_{n}(z)\right| \leqq A_{p} \delta^{-2 / p}$ for $|y| \leqq \pi / 2-\delta$. Hence the $G_{n}(0)(n=1,2, \cdots)$ are bounded; hence there is a positive constant $B_{0}$ such that $H_{n}(0) \geqq B_{0}$, since $H_{n}(0)=|F(0)|\left|G_{n}(0)\right|^{-1}$. Thus we have proved the existence of the Blaschke function $H(z)$ in $S$. Taking $G(z)=F(z) / H(z)$, the proof for the case $F(0) \neq 0$ can be completed exactly as the Riesz proof. Let now $F(z)$ have a zero of order $m$ at the origin. Taking $H(z)=H_{0}(z)\{\tanh (z / 2)\}^{m}$, where $H_{0}(z)$ is the Blaschke function belonging to $F(z)(\tanh (z / 2))^{-m}$, we have proved the theorem except for its last assertion. Now we have to anticipate (b) and (d). We replace $F(z)$ by $F_{0}(z)=F(z)$ exp (icz) and $G(z)$ by $G_{0}(z)$ $=G(z) \exp (i c z)$, fixing $c$ so that $\left|F_{0}(x-i \pi / 2)\right|_{p}=\left|F_{0}(x+i \pi / 2)\right|_{p}$, while $H(z)$ is not altered. Using (5.11) and (7.28) and the inequality $\left|G_{0}(z)\right| \geqq\left|F_{0}(z)\right|$ $(|y|<\pi / 2)$, we deduce that, for almost all $x$ in $(-\infty, \infty),|H(x \pm i \pi / 2)|=1$, which completes the proof.

7.3. Proof of (b)-(f). The function $\psi(z)=\{G(z)\}^{p / 2}(0<p<\infty)$ can be defined uniquely in $S$; it belongs to $S_{2}(-\pi / 2, \pi / 2)$. Therefore, by the Titchmarch theorem, its limit-functions $(y \downarrow a, y \uparrow b)$ exist. The corresponding result holds for the function $H(z)$ which belongs to $S_{\infty}(-\pi / 2, \pi / 2)$. Thus we have proved the existence of the limit-functions of $F(z)$ for normal approach to the lines $z=x \pm i \pi / 2$. We omit the proof of the more general assertion of the theorem.

Proof of (c). Let $E$ be a measurable set in $(-\infty, \infty)$ of finite or infinite measure; let $0<p<\infty$. Following the Riesz method, we can show that:

$$
\int_{E}|F(x+i y)|^{p} d x \rightarrow \int_{E}|F(x \pm i \pi / 2)|^{p} d x \quad(0<p<\infty)
$$

as $y \downarrow-\pi / 2$ or $y \uparrow \pi / 2$, respectively, and can then deduce (c) from (7.31) by means of the Egoroff theorem, taking some care as the interval is infinite. To prove (d) we apply the Hardy-Ingham-Pólya theorem to the function $F(z)$ in the closed strip $|y| \leqq \eta=\pi / 2-\delta$; we have, for $0<p<\infty$,

$$
|F(x+i y)|_{p} \leqq|F(x-i \eta)|_{p}^{(y-\eta) /(2 \eta)}|F(x+i \eta)|_{p}^{(y+\eta) /(2 \eta)} .
$$

(22) Loc. cit. (4.13). 
Taking $\eta \rightarrow \pi / 2$ and using (5.21) we deduce (d). The assertion (e) is proved by a customary argument, using (5.11), Lemma 12 and (c). By a similar argument it can be shown that the right side of (5.23) vanishes identically for $y<-\pi / 2$ and $y>\pi / 2$. This, together with (e), gives (f) for $1 \leqq p<\infty$; when $p=\infty$ then we apply (f) to the function $F(z) \exp \left(-\delta z^{2}\right)$ and take $\delta \rightarrow 0$. The first part of $\left(c^{\prime}\right)$ is deduced by means of the conformal transformation (7.21) and the Egoroff theorem, the second and third part of $\left(c^{\prime}\right)$ are deduced by Lemma $12^{\prime}$, taking $n=1$, and by the Poisson integral. Thus we have proved the theorem.

7.4. Consequences of Theorem 13. The following results can be deduced from Theorem 13.

Theorem 14(23). Let $F(z) \in S_{p}(a, b)$ and $F(x+i a) \in L_{q}(-\infty, \infty), F(x+i b)$ $\in L_{r}(-\infty, \infty)$. If $0<q \leqq r \leqq p \leqq \infty$, then $F(z)$ belongs to $S_{s}(a, b)$ whenever $r \leqq s \leqq p$. If $0<p \leqq q \leqq r \leqq \infty$, then $F(z)$ belongs to $S_{s}(a, b)$ whenever $p \leqq s \leqq q$. The corresponding results hold for $r \leqq q \leqq p$ and $p \leqq r \leqq q$.

THEOREM 15(24). Let the function $\left(1-w^{2}\right)^{-1 / p}$ be defined so that it is analytic for $|w|<1$, and let $F(z)=f(w)$ where $w=\tanh (z / 2)$. Then $F(z)$ belongs to $S_{p}(-\pi / 2, \pi / 2)$ if and only if $\left(1-w^{2}\right)^{-1 / p} f(w)$ belongs to the Riesz class $H_{p}$ $(0<p \leqq \infty)$.

TheOREM 13'. Let (i) $F(z)$ be analytic in $S(a, b)$, (ii) $0<k<\pi(b-a)^{-1}$, $0<p<\infty$ and $\int_{-x}^{x}|F(\xi+i y)|^{p} d \xi<A \exp \left(e^{k|x|}\right)$ for $-\infty<x<\infty, a<y<b$. Then Theorem 13(a, b) holds, replacing the assertion $G(z) \in S_{p}(a, b)$ by the weaker one: $G(z)$ is analytic in $S$.

THEOREM 13". If $F(z)$ satisfies (i) and (ii), and if (iii) its limit functions $F(x+i a)$ and $F(x+i b)$ belong to $L_{p}(-\infty, \infty)$, then $F(z) \in S_{p}(a, b)$.

The latter result has been proved by Hardy, Ingham and Pólya $\left({ }^{25}\right)$, replacing (ii) by the slightly stronger condition $(2 x)^{-1} \int_{-x}^{x}|F(\xi+i y)|{ }^{p} d \xi$ $<A \exp \left(e^{k|x|}\right)$ and adding the condition that $|F(z)|$ should be continuous in any finite part of the closed strip $a \leqq y \leqq b$.

To prove Theorem $13^{\prime}$ we show that the function $Q(z)=F(z) \exp$ $\left\{-\eta \cosh k^{\prime}(z-(a+b) / 2)\right\} \quad\left(\eta<0, k<k^{\prime}<\pi(b-a)^{-1}\right)$ belongs to $S_{p}(a, b)$, using integration by parts, and apply Theorem $13(\mathrm{a}, \mathrm{b})$.

Therefore $F(x+i a)$ and $F(x+i b)$ exist. Now we apply (5.22) to $Q(z)$ and take $\eta \rightarrow 0$, which proves Theorem $13^{\prime \prime}$.

7.5. Proof of Lemma 11. Without loss of generality, we may take $z_{0}=i b$ where $b>0$. The linear transformation

(23) Compare V. Smirnoff, C. R. Acad. Sci. Paris vol. 188 (1929) pp. 131-133.

${ }^{(24)}$ Compare Hille-Tamarkin, loc. cit., Lemma 2.5, and H. Kober, Bull. Amer. Math. Soc. vol. 49 (1943) pp. 437-443, Lemma 2. The proof of the present result is similar, but more difficult.

(25) Loc. cit., see Theorems 1, 7, 9 and 10. 


$$
w=(z+i b) /(i b-z), \quad z=i b(w-1) /(w+1)
$$

maps the lower half-plane on the interior of the unit circle $\Gamma$ and the point $z_{0}$ on $w=\infty$, the point $z=\infty$ on $w=-1$, the real axis on $\Gamma$. The function $h(w)=F(z)=F\left\{i b(w-1)(w+1)^{-1}\right\}$ is analytic and bounded in the interior of $\Gamma$. Let $E$ be the set on which the limit function $h\left(e^{i \theta}\right)$ of $h(w)\left(w \rightarrow e^{i \theta}\right.$, $-\pi<\Theta<\pi$ ) exists, let

$$
\begin{aligned}
h(w)=\sum_{k=0}^{\infty} a_{k} w^{k}, \quad s_{m}(w)=\frac{1}{m+1} \sum_{j=0}^{m} \sum_{k=0}^{j} a_{k} w^{k} \\
\quad(|w|<1, m=0,1,2, \cdots),
\end{aligned}
$$

and let $0<R_{0}<R_{1}<R_{2}<\cdots<1$ and $R_{n} \rightarrow 1$ as $n \rightarrow \infty$.

To any $m(m=0,1,2, \cdots)$ we can find an integer $k_{m}$ such that, uniformly for $|w| \leqq 1$,

$$
\left|h\left(R_{m} w\right)-s_{k_{m}}\left(R_{m} w\right)\right| \leqq 1 /(m+1) .
$$

Thus we have a sequence of polynomials

$$
H_{m}(w)=s_{k_{m}}\left(R_{m} w\right) \quad(m=0,1,2, \cdots) .
$$

At any point of $E, H_{m}\left(e^{i \Theta}\right)$ tends to $h\left(e^{i \Theta}\right)$ as $m \rightarrow \infty$. For, given $\epsilon>0$, at this point we have $\left|h\left(R_{m} e^{i \Theta}\right)-h\left(e^{i \Theta}\right)\right| \leqq \epsilon / 2$ for $m \geqq \mathrm{M}$ and, using (7.53), $\mid H_{m}\left(e^{i \Theta}\right)$ $-h\left(R_{m} e^{i \theta}\right) \mid \leqq \epsilon / 2$ for all sufficiently large values of $m\left(m \geqq 2 \epsilon^{-1}-1\right)$. Given $A<1$, we have $h\left(R_{m} w\right)-h(w) \rightarrow 0$, as $m \rightarrow \infty$, uniformly for $|w| \leqq A$. Therefore, by (7.53), $H_{m}(w) \rightarrow h(w)$, as $m \rightarrow \infty$, uniformly for $|w| \leqq A$.

Finally we show that $\left|H_{m}(w)\right| \leqq|F(x)|_{\infty}$ for $|w|<1$. The $s_{m}(w)$ are the Cesàro means of the partial sums of the power series of $h(w)$. This function is bounded in $\Gamma$; therefore $\left.{ }^{26}\right)$, for $|w|<1$, we have $\left|s_{k_{m}}(w)\right| \leqq$ ess.u.b. $\left|h\left(e^{i \theta}\right)\right|$ $=|F(x)|_{\infty}$ and, a fortiori, $\left|H_{m}(w)\right| \leqq|F(x)|_{\infty}$ for $|w| \leqq 1$.

Hence the rational functions $r_{n}(z)=H_{n}\left\{(i b+z)(i b-z)^{-1}\right\}$ possess all the properties required in the lemma.

${ }^{(26)}$ By a result due to J. F. Steffensen. See, for instance, E. Landau, Darstellung und Begründung einiger neuerer Ergebnisse der Funktionentheorie, Berlin, 1916, first theorem.

The UnIVERSITY,

Edgbaston, Birmingham, England. 